\title{
Cuidado de la salud como determinante del consumo de alimentos orgánicos en Chile
}

\author{
Health care as a determinant of the consumption of organic foods in Chile
}

Sebastián Araya-Pizarro*1, Luperfina Rojas-Escobar ${ }^{1}$

\begin{abstract}
RESUMEN
La investigación tuvo como objetivo determinar la influencia del cuidado de la salud en la frecuencia de consumo de alimentos orgánicos, un mercado de promisorio crecimiento en Chile. El estudio se llevó a cabo utilizando una encuesta presencial aplicada a 384 consumidores chilenos (Región de Coquimbo) bajo un muestreo por conveniencia. Los datos obtenidos se analizaron con un modelo multivariante de regresión logística binaria y pruebas de asociación chi-cuadrado de Pearson. Los resultados evidenciaron que los productos orgánicos son percibidos como más saludables y de mejor calidad que los productos convencionales, y que las personas de mayor nivel socioeconómico (educación y empleo) y que demuestran interés por el cuidado de la salud tienen probabilidades más altas de comprarlos regularmente. Además, se reveló que es fundamental para el éxito comercial del alimento orgánico que sus vendedores se preocupen de contar con un etiquetado adecuado (información nutricional) y productos certificados (sello orgánico).
\end{abstract}

Palabras clave: alimentos orgánicos, preferencias del consumidor, regresión logística binaria, cuidado de la salud, Chile.

\begin{abstract}
The research objective was to determine the influence of health care on the frequency of consumption of organic foods, a market with promising growth in Chile. The study was carried out using a face-to-face survey applied to a sample of 384 Chilean consumers (Coquimbo Region) under a convenience sample. The data obtained were analyzed using a multivariate binary logistic regression model and Pearson chi-square association tests. The results showed that organic products are perceived as healthier and better quality than conventional products, and that people of higher socioeconomic status (education and employment) and who show an interest in health care have a higher probability of buying them regularly. Also, it was revealed that it is essential for the commercial success of organic food that its sellers worry about having an adequate labeling (nutritional information) and certified products (organic certification).
\end{abstract}

Keywords: organic foods, consumer preferences, binary logistic regression, health care, Chile.

\section{Introducción}

El mercado de productos orgánicos en el mundo se encuentra en expansión, y destaca en especial el crecimiento de la participación de la agricultura de productos orgánicos, cuyos principales representantes son Estados Unidos, Alemania y Francia (Aslihan Nasir y Karakaya, 2014). En Chile, se trata de un sector promisorio, que lentamente ha ido adquiriendo una relativa importancia en la industria orgánica mundial. Las exportaciones chilenas, por ejemplo, pasaron de US\$ 50 millones en 2009 a US\$ 280 millones en
2018. Mientras que las importaciones de alimentos orgánicos en el país crecieron en un $60 \%$, pasando de US\$ 9.9 millones a casi US\$ 16 millones durante el periodo 2015-2016 (Ibáñez, 2019; Díaz, 2017).

Sumado a lo anterior, se observa el interés creciente del consumidor por adquirir productos ecológicos, sociales y orientados al cuidado de la salud, lo que ha presionado el surgimiento de una gama de productos alimenticios bajo distintas certificaciones y denominaciones tales como comercio justo, empresa B, kosher, libre de gluten, natural y, por supuesto, alimento orgánico. Cabe indicar que se considera un producto orgánico

\footnotetext{
1 Facultad de Ciencias Sociales, Empresariales y Jurídicas de la Universidad de La Serena, Amunátegui s/n, La Serena, Chile.

* Autor para correspondencia: saraya@userena.cl
}

Fecha de Recepción: 8 de julio, 2019.

Fecha de Aceptación: 1 de agosto, 2020. 
o ecológico o biológico aquel alimento que se consigue sin la intervención de agentes químicos (fertilizantes, herbicidas o pesticidas) en todas las etapas de su producción y en los suelos donde es cultivado (Higuchi, 2015).

Esta popularidad ha llevado a que los productos orgánicos sean objeto de análisis permanente en el ámbito académico, desde diferentes perspectivas. Así, sobresalen investigaciones respecto al perfil de los consumidores de productos orgánicos y sus motivaciones de compra, el examen de los factores que impulsan las compras orgánicas, la disposición a pagar por los productos orgánicos, los principales obstáculos y tendencias de compra y el valor de la información para quienes adquieren este tipo de productos (Andrade y Ayaviri, 2018; Stoleru et al., 2019). Sin embargo, y a pesar de la considerable evidencia, los determinantes del comportamiento de compra orgánico siguen siendo inciertos, y quedan sujetos a variables de tipo contextual y delimitadas a la percepción manifiesta de los propios consumidores.

En tal contexto surge la idea de estudiar la influencia que tiene la preocupación del consumidor por su salud sobre la frecuencia de compra de alimentos orgánicos, atendiendo al potencial que reviste la demanda de un segmento creciente en Chile y que de acuerdo a los últimos antecedentes responde a un fenómeno mundial, que reconoce un consumidor más preocupado por el cuidado de la salud, el medio ambiente y las prácticas de responsabilidad social (Cea-Valencia et al., 2016).

Este artículo se estructura en cuatro acápites. Primeramente, expone una breve revisión teórica sobre el comportamiento del consumidor de alimentos orgánicos. Continúa con la descripción del marco metodológico de la investigación. Luego, sintetiza los resultados encontrados. Y, por último, se muestran las principales conclusiones.

\section{Revisión teórica}

En Chile, como en muchos otros países del mundo, la demanda de alimentos orgánicos ha crecido notablemente durante las últimas décadas (Díaz, 2017; Ditlevsen et al., 2019; Aslihan Nasir y Karakaya, 2014). En general se sostiene que el desarrollo de la producción moderna de alimentos orgánicos ha sido impulsado en gran medida por las preocupaciones ambientales, el cuidado de la salud humana y la seguridad alimentaria, pues los alimentos orgánicos se describen con frecuencia como más saludables que los alimentos convencionales (Bryła, 2016; Rana y Paul, 2017). Por ello, no sorprende que en años recientes se observe un aumento de las investigaciones orientadas a conocer el comportamiento y actitudes del consumidor en relación con este tipo de productos, $\mathrm{y}$ en especial sobre los factores que determinan su elección de compra (Bryła, 2016; Ditlevsen et al., 2019; Janssen, 2018; Massey et al., 2018; Ozguven, 2012; Rana y Paul, 2017; Singh y Verma, 2018). Su adecuada comprensión permitiría consumar el potencial de los alimentos orgánicos de convertirse en un mercado verdaderamente dominante.

La literatura revela que dentro de los determinantes del consumo de productos orgánicos se encuentra la percepción que tienen los consumidores de que los alimentos orgánicos son más saludables y nutritivos que los alimentos convencionales, dado que se producen naturalmente con menos o ningún producto químico (Stoleru et al., 2019). Esta percepción lleva a evaluaciones más positivas de los alimentos orgánicos y mayor motivación para comprarlos (Hwang y Chung, 2019). Por tanto, la preocupación y conciencia por la salud se transforma en un determinante clave que impacta significativamente en la intención de compra de alimentos orgánicos (Singh y Verma, 2018).

Aslihan Nasir y Karakaya (2014), por ejemplo, demostraron que los consumidores con actitudes más favorables hacia los alimentos orgánicos son aquellos que poseen niveles más altos de orientación hacia la salud y un comportamiento de consumo socialmente responsable. En la misma línea, Rana y Paul (2017) hallaron que los consumidores con mayor conciencia de su salud tienen una mayor preferencia por alimentos orgánicos que por los alimentos cultivados de manera convencional. Explican que este cambio en la actitud de los consumidores modernos está muy influenciado por la creciente incidencia de enfermedades relacionadas con el estilo de vida, como los trastornos cardíacos y la depresión. Esto demuestra que el mercado guía sus decisiones de acuerdo a los beneficios superiores percibidos de los alimentos orgánicos (Massey et al., 2018). Bryła (2016) postula que los consumidores están convencidos de que los alimentos orgánicos son más caros, más saludables, más respetuosos con el medio ambiente, de mejor sabor y más auténticos que los alimentos convencionales. Por 
tanto, los atributos que determinan la preferencia por los productos orgánicos corresponden a la salubridad y la alta calidad que los caracterizan (Janssen, 2018).

Respecto a la noción de salud asociada a los alimentos orgánicos, Ditlevsen et al. (2019) desarrollaron un interesante estudio en el que analizaron tres conceptos de salud con los que los consumidores explican sus preferencias por los productos orgánicos: salud como valor nutricional (mejor composición nutricional), salud como pureza (ausencia de aditivos alimentarios, conservantes y residuos) y salud como placer (disfrutar de productos de mejor sabor, textura u otras características sensoriales superiores). La salud como pureza es la consideración dominante que explica por qué adquieren productos alimenticios orgánicos.

También es posible hallar artículos donde se examinan características demográficas y socioeconómicas que influyen en las preferencias por productos orgánicos, pero sin resultados concluyentes. Por ejemplo, algunos estudios revelarían que las mujeres son más propensas a comprar y consumir alimentos orgánicos en grandes cantidades, y que tienen actitudes más positivas hacia estos que los hombres (Ureña et al., 2008). Otras investigaciones demuestran que los compradores de alimentos orgánicos exhiben niveles de ingresos más altos y mayor nivel educativo que aquellos que no son compradores (Higuchi, 2015).

Finalmente, existen trabajos que han analizado el comportamiento del consumidor con respecto a cómo la información que se entrega del producto afecta su elección de compra, y se encontró que los niveles más altos de conocimientos están asociados con la razón de la selección (Stanton y Cook, 2019). Se advierte entonces que es necesario informar sobre la calidad de los alimentos, de manera de entregar credibilidad y confianza a sus consumidores. Como señalan Nandonde y Kuada (2016), los consumidores no evalúan la calidad solo por las características físicas del producto, sino que necesitan confirmar sus estándares y precisamente la certificación orgánica, como símbolo de agricultura sostenible y vida saludable, constituye un buen indicio de la calidad del alimento.

Lo anterior evidencia que informar sobre las ventajas y beneficios que tiene la producción y consumo de alimentos orgánicos es útil para desarrollar la demanda de estos productos, especialmente en la creación de estrategias de comunicación para su posicionamiento efectivo en mercados emergentes como el de Chile (Sweeney et al., 2015).

\section{Metodología}

La investigación, de tipo cuantitativa y corte transversal, se realizó en la Región de Coquimbo (Chile), sobre una muestra de 384 personas consumidoras de productos orgánicos a través de un muestreo por conveniencia. El tamaño de la muestra se determinó utilizando un nivel de confianza del $95 \%$, un error muestral de $5 \%$, una proporción del $50 \%$ y una población infinita ( $\mathrm{N}>30.000$ personas).

Los datos se obtuvieron mediante una encuesta presencial ad-hoc que fue aplicada en supermercados y tiendas especializadas del rubro en las ciudades de La Serena y Ovalle (siete centros de recolección). Las preguntas del cuestionario (13 ítems) se construyeron bajo una escala Likert de 5 puntos, siendo: $1=$ muy en desacuerdo, $2=$ en desacuerdo, 3 = indiferente, $4=$ de acuerdo y $5=$ muy de acuerdo. La fiabilidad del instrumento se midió a través de cómputo del alfa de Cronbach, que evidenció una buena consistencia de la escala utilizada $(\mathrm{alfa}=0,91)$. Además, se incluyó un grupo de variables demográficas y socioeconómicas, que podrían influir en las preferencias por alimentos orgánicos, y que son las siguientes:

- Sexo: hombre (1) y mujer (2).

- Edad: 18-35 años (1), 36-45 años (2), 46-55 años (3), 56-65 años (4) y más de 65 (5).

- Estado civil: soltero/a (1), casado/a (2), separado/a (3) y viudo/a (4).

- Ocupación: empleado (1), independiente (2), estudiante (3) y ama de casa (4).

- Nivel de estudios: técnico (1), profesional (2), universitario (3) y postgrado (4).

- Estrato económico: USD 2.001 o más (1), USD 1.201-2.000 (2) y USD 750-1.200 (3).

El análisis de la información recolectada se procesó mediante un modelo de regresión logístico binario compuesto solo de variables categóricas. El modelo de regresión logística se define como una técnica estadística multivariante que utiliza una variable de criterio dicotómica y una o más variables predictoras cualitativas, ordinales o cuantitativas. 
En concreto, en este estudio se buscó determinar los factores que incrementan la probabilidad de consumir frecuentemente alimentos orgánicos.

De este modo se clasificó como variable dependiente del modelo la frecuencia de compra de alimentos orgánicos, pudiendo ser habitual (al menos una vez a la semana) o no. La codificación de las variables independientes fue realizada en una categorización binaria a partir de las preguntas del cuestionario y las variables demográficas y socioeconómicas del consumidor, tal como se aprecia en la Tabla 1.

El modelo de regresión logístico se diseñó bajo la siguiente estructura matemática:

$$
\log \left(\frac{p}{1-p}\right)=b_{0}+b_{1} x_{1}+b_{2} x_{2}+\ldots+b_{k} x_{k}
$$

Donde $p$ representa la probabilidad de que el comprador consuma frecuentemente alimentos orgánicos (situación de interés). Según el valor de las variables explicativas se puede estimar directamente la probabilidad de que ocurra el evento de interés del modo siguiente:

$$
\hat{p}=\left(\frac{e^{\hat{b}_{0}+\hat{b}_{1} x_{1}+\hat{b}_{2} x_{2}+\ldots+\hat{b}_{k} x_{k}}}{1-e^{\hat{b}_{0}+\hat{b}_{1} x_{1}+\hat{b}_{2} x_{2}+\ldots+\hat{b}_{k} x_{k}}}\right)
$$

Finalmente, cabe destacar que para establecer el poder predictivo y ajuste del modelo se desarrolló la prueba omnibus sobre los coeficientes del modelo, la prueba de Hosmeer \& Lemeshow y el $R$ cuadrado de Nagelkerke.

\section{Resultados}

Este apartado se divide en tres secciones: I) caracterización de la muestra, II) asociación entre variables categóricas, y III) regresión logística.

\section{Caracterización de la muestra}

La Tabla 2 muestra que del total de consumidores encuestados, el 49,5\% son mujeres y 50,5\% hombres. La mayoría es menor de 46 años $(80,2 \%)$, tiene estudios de licenciatura $(68,8 \%)$, trabaja como empleado dependiente (80,2\%), de estado civil soltero $(68,8 \%)$ y pertenece al estrato socioeconómico medio típico $(53,4 \%)$.

\section{Asociación entre variables categóricas}

La Tabla 3 sintetiza el análisis de las variables categóricas del modelo. En ella se aprecia la existencia de una asociación significativa entre la frecuencia de compra y ocho variables independientes. En relación con las variables personales, solo resultaron relevantes variables socioeconómicas, es decir, la ocupación, el nivel de estudios y los ingresos (estrato social). Ello deja de manifiesto que los consumidores frecuentes de productos orgánicos corresponden mayormente a una clase media emergente, que se caracteriza por contar con niveles superiores de estudio y una situación laboral estable. Así se confirma lo que otros autores han destacado del perfil del consumidor de productos orgánicos (Díaz et al., 2015; Lutterbeck, 2001). Al respecto, es importante señalar el gran porcentaje de personas de clase media típica que consumen

Tabla 1. Codificaciones de variables categóricas.

\begin{tabular}{rll}
\hline $\mathrm{N}^{\circ}$ & \multicolumn{1}{c}{ Variable categórica } & \multicolumn{1}{c}{ Codificación de parámetro } \\
\hline 1 & Situación económica & 1: favorable (267); 0: no favorable (117) \\
2 & Edad & 1: 35 años o menos (211); 0: mayor a 35 años (173) \\
3 & Estado civil & 1: soltero (264); 0: no soltero (120) \\
4 & Ocupación & 1: empleado (308); 0: no empleado (76) \\
5 & Nivel de estudios & 1: profesional (273); 0: no profesional (111) \\
6 & Sexo & 1: mujer (190); 0: hombre (194) \\
7 & Motivo: cuidado de la salud & 1: Sí (317); 0: No (67) \\
8 & Motivo: calidad del producto & 1: Sí (333); 0: No (51) \\
9 & Motivo: precio económico & 1: Sí (275); 0: No (109) \\
10 & Producto certificado & 1: Sí (256); 0: No (128) \\
11 & Producto con información nutricional & 1: Sí (292); 0: No (92) \\
12 & Frecuencia habitual de compra & 1: Sí (235); 0: No (149) \\
\hline
\end{tabular}


Tabla 2. Frecuencias absolutas y relativas

de las variables demográficas y socioeconómicas.

\begin{tabular}{|c|c|c|c|c|c|c|}
\hline $\mathrm{N}^{\mathrm{o}}$ & Dimensión & & & Jiveles & & \\
\hline & Sexo & Hombre & Mujer & & & \\
\hline 1 & Total casos & 194 & 190 & & & \\
\hline & Frecuencia $(\%)$ & 50,5 & 49,5 & & & \\
\hline & Edad (años) & $18-35$ & $36-45$ & $46-55$ & $56-65$ & $>65$ \\
\hline 2 & Total casos & 211 & 97 & 52 & 18 & 6 \\
\hline & Frecuencia $(\%)$ & 54,9 & 25,3 & 13,5 & 4,7 & 1,6 \\
\hline & Estudios & Técnico & Bachillerato & Licenciado & Postgrado & \\
\hline & Total casos & 91 & 20 & 264 & 9 & \\
\hline & Frecuencia (\%) & 23,7 & 5,2 & 68,8 & 2,3 & \\
\hline & Ocupación & Empleado & Independiente & Estudiante & Amo de casa & \\
\hline 4 & Total casos & 308 & 51 & 16 & 9 & \\
\hline & Frecuencia $(\%)$ & 80,2 & 13,3 & 4,2 & 2,3 & \\
\hline & Estado civil & Soltero & Casado & Separado & Viudo & \\
\hline 5 & Total casos & 264 & 95 & 18 & 7 & \\
\hline & Frecuencia (\%) & 68,8 & 24,7 & 4,7 & 1,8 & \\
\hline & Nivel de ingresos & C1 (alta) & C2 (típica) & C3 (baja) & & \\
\hline 6 & Total casos & 62 & 205 & 117 & & \\
\hline & Frecuencia (\%) & 16,1 & 53,4 & 30,5 & & \\
\hline
\end{tabular}

Tabla 3. Pruebas de asociación entre variables del modelo y la frecuencia de compra.

\begin{tabular}{lcl}
\hline Variables & Puntuación & Significancia \\
\hline Sexo & 1,437 & 0,231 \\
\hline Edad & 0,809 & 0,937 \\
\hline Estado civil & 1,025 & 0,795 \\
\hline Nivel de ingresos & 7,636 & $0,022^{* *}$ \\
\hline Ocupación & 6,463 & $0,091^{*}$ \\
\hline Nivel de estudios & 18,330 & $0,000^{* * *}$ \\
\hline Motivo: calidad del producto & 23,928 & $0,000^{* * *}$ \\
\hline Motivo: cuidado de la salud & 57,798 & $0,000^{* * * *}$ \\
\hline Motivo: precio económico & 15,103 & $0,004 * * *$ \\
\hline Producto certificado & 31,884 & $0,000^{* * *}$ \\
\hline Producto con información nutricional & 47,244 & $0,000^{* * *}$ \\
\hline
\end{tabular}

* Significativo al 10\%, ** Significativo al 5\%, *** Significativo al $1 \%$. frecuentemente este tipo de alimentos y que podría explicarse por lo que Lutterbeck (2001) denomina el segmento de consumo clásico. Esto es, un grupo de consumidores que a pesar de tener ingresos más bajos mantienen un compromiso con los productos ecológicos como parte de su estilo de vida.

Los resultados también revelaron una asociación significativa entre la frecuencia y las motivaciones de compra vinculadas al cuidado de la salud, la calidad del producto y los precios convenientes. En otras palabras, el consumidor de alimentos orgánicos sería, como señala Higuchi (2015), un tipo de consumidor Lohas (del inglés lifestyles of health and sustainability), es decir, que exhibe un estilo de vida sano y sostenible y sensible al precio, y cuya principal motivación de compra son sus creencias sobre la salud, la calidad, la sostenibilidad y lo natural y nutritivo del producto (Hwang y Chung, 2019). En ese sentido, diversos trabajos han expuesto al precio como una barrera que limita la compra en mayor cuantía y frecuencia 
de los alimentos orgánicos, habitualmente más caros que un producto convencional (Nasir y Karakaya, 2014; Stoleru et al., 2019). Por tanto, el consumidor de alimentos orgánicos es sensible al precio, lo que implica que, aunque muestra disposición a pagar un sobreprecio por adquirir este tipo de productos (Andrade y Ayaviri, 2018), su decisión final queda circunscrita a aquella opción que presenta el binomio precio-calidad más conveniente (económica).

En cuanto a las características comerciales del producto orgánico que resultan determinantes en su selección, destacan la información nutricional del alimento y la certificación orgánica (Díaz et al., 2015; Stanton y Cook, 2019), lo que deviene en respuesta a una mayor preocupación de la población por garantizar la inocuidad y calidad de los alimentos que consume (Auriol y Schilizzi, 2015). Así, tanto el etiquetado nutricional como la certificación se transforman en los medios tradicionales por los cuales los productores pueden suministrar una medida evidenciable del estándar de su oferta comercial (Parga-Dans y Alonso, 2017).

\section{Regresión logística}

La Tabla 4 resume los principales resultados de la regresión logística binomial aplicada.

El análisis de los factores que afectan la frecuencia de compra de los productos orgánicos indicó que las variables nivel de estudios, cuidado de la salud (motivo de compra) e información nutricional (etiqueta) presentan significancia estadística a un nivel $p$ de $1 \%$, mientras que la ocupación mostró significancia a un nivel de $p$ de 5\%.

El factor con mayor poder de predicción fue el motivo de compra denominado "cuidado de la salud". Ello confirma que aquellas personas que tienen como motivación consumir alimentos naturales libres de químicos, con características nutricionales superiores e inocuos para la salud, entre otros, buscarán adquirir de forma habitual productos orgánicos $\mathrm{y}$, por consiguiente, mostrarán una frecuencia de consumo mayor. En resumen, la probabilidad de consumir alimentos orgánicos aumenta en casi 4 veces a medida que existe un consumidor preocupado por el cuidado de su salud. En concordancia con lo expuesto en otros trabajos, se comprueba que la salud motiva a comprar alimentos orgánicos (Ozguven, 2012), porque los métodos de producción ecológicos son percibidos como más saludables y de calidad superior a los utilizados por la agricultura convencional.

Otro factor con similar capacidad de predicción es la información nutricional del producto. Esto implica que los consumidores repiten la compra cuando el alimento orgánico cuenta con información que especifica su composición y beneficios nutricionales. Estos resultados concuerdan con estudios previos que han revelado

Tabla 4. Regresión logística binaria con las variables del modelo.

\begin{tabular}{lccccc}
\hline Variable & $\beta$ & E.T. & Wald & Sig. & $\operatorname{Exp}(\beta)$ \\
\hline Sexo & 0,301 & 0,241 & 1,562 & 0,211 & 1,351 \\
Edad & $-0,078$ & 0,253 & 0,096 & 0,757 & 0,925 \\
Estado civil & 0,104 & 0,270 & 0,148 & 0,700 & 1,110 \\
Ocupación & 0,759 & 0,294 & 6,680 & $0,010^{*}$ & 2,135 \\
Nivel de estudios & 0,960 & 0,262 & 13,388 & $0,000^{* *}$ & 2,611 \\
Situación económica & 0,416 & 0,259 & 2,568 & 0,109 & 1,516 \\
Motivo: cuidado de la salud & 1,321 & 0,349 & 14,327 & $0,000^{* *}$ & 3,747 \\
Motivo: calidad del producto & 0,205 & 0,252 & 0,661 & 0,416 & 1,227 \\
Motivo: precio económico & 0,316 & 0,274 & 1,328 & 0,249 & 1,371 \\
Producto certificado & $-0,100$ & 0,280 & 0,128 & 0,721 & 0,905 \\
Producto con información nutricional & 1,309 & 0,312 & 17,575 & $0,000^{* *}$ & 3,703 \\
Constante & $-3,620$ & 0,603 & 36,028 & $0,000^{* *}$ & 0,027 \\
Prueba de Hosmer y Lemeshow & $\chi^{2}=8,573$ & Sig. $=0,38$ & & & \\
R $^{2}$ de Cox y Snell & 0,207 & & & & \\
R de Nagelkerke & 0,280 & & & & Lím. inferior \\
Curva COR & Área & E.T. & Sig. & símerior \\
& 0,747 & 0,026 & $0,000^{* *}$ & 0,696 & 0,799 \\
\hline
\end{tabular}

* Significativo al 5\%,**Significativo al $1 \%$. 
que el etiquetado nutrimental del producto es una variable significativa que impacta en la selección y decisión de compra de alimentos (López-Cano y Restrepo-Mesa, 2014). Por ende, los productores tendrían mayor éxito comercial en la medida que su oferta demuestre un nivel informativo satisfactorio, que entregue seguridad al sujeto sobre lo que está consumiendo.

El resto de los factores significativos corresponden a variables socioeconómicaso. Los hallazgos indican que una persona con un nivel de estudios alto y que trabaja de forma estable tiene mayor probabilidad de consumir habitualmente productos orgánicos. Estos resultados guardan relación con lo investigado por Vasco et al. (2015), quienes sostienen que la frecuencia de compra está vinculada con el perfil socioeconómico del sujeto. Específicamente señalaron que las personas que poseen un mayor nivel de escolaridad, así como más ingresos económicos, gastan más en el consumo de productos orgánicos y ecológicos. Cabe destacar que la variable situación socioeconómica no resultó significativa para el modelo predictivo de esta investigación $(p=0.11)$, lo que apoyaría lo asegurado por Lutterbeck (2001) en cuanto a que los consumidores de orgánicos se encuentran fragmentados en diferentes grupos de ingreso, de acuerdo con el grado de compromiso demostrado por sus motivaciones de consumo (cuidado de la salud y estilo de vida). Ello supondría que la relación entre el nivel de ingresos y la frecuencia de compra no sea tan marcada.

En síntesis, los resultados permiten constatar que el consumo de alimentos orgánicos se explica mayormente por motivos del cuidado de la salud, por lo que es recomendable tomar esto en cuenta para que se comuniquen y promocionen los beneficios derivados de su elección. La calidad del producto (vinculada a las características nutricionales) y la certificación son variables esenciales para el consumidor, por lo que es necesario que la presentación de los alimentos incorpore dicha información que respalde y dé garantías de su composición. Otro factor al que el consumidor da bastante importancia es el precio, por lo que las empresas deben incrementar sus esfuerzos por generar productos con precios competitivos.

Por último, es importante mencionar que además de aplicar la prueba de Hosmer y Lemeshow, que determinó un ajuste adecuado del modelo $(p>0,05)$, y computar el coeficiente $R^{2}$ de Nagelkerke, que demostró que el modelo de regresión logística explica aproximadamente el $28 \%$ de la variable endógena, se realizó una prueba de discriminación del modelo para evaluar el grado en que este distingue entre individuos que consumen frecuentemente alimentos orgánicos y los que no. Para ello se realizó el análisis ROC, prueba construida para la probabilidad estimada. El área bajo la curva ROC mostró resultados satisfactorios $(0,747)$, la sensibilidad fue de 0,708 y la especificidad de 0,436 , implicando que quienes buscan cuidar su salud, tienen alto nivel de estudio, poseen empleo estable y prefieren productos que declaren su información nutrimental, cuentan con mayores probabilidades de consumir habitualmente productos ecológicos.

\section{Conclusiones}

Del análisis de los factores que impulsan el consumo de alimentos orgánicos en Chile se concluye que existe una asociación significativa entre la frecuencia y las motivaciones de compra vinculadas al cuidado de la salud, la calidad del producto y los precios convenientes. De este modo el consumidor de alimentos orgánicos se caracteriza por tener un estilo de vida saludable, ser sensible al precio y percibir que los productos ecológicos son de mayor calidad que los convencionales.

Se infiere también que las variables socioeconómicas educación, ocupación y nivel de ingresos influyen en el consumo de alimentos orgánicos en una relación directa, y que las características comerciales del producto que resultan determinantes en su selección son la información nutricional y la certificación orgánica. Así, tanto el etiquetado como la certificación se convierten en los medios básicos por los cuales los productores pueden suministrar una medida evidenciable del estándar de su oferta comercial.

Por otra parte, se confirma que los atributos cuidado de la salud, nivel de educación, ocupación e información nutricional del producto son predictores en la frecuencia de compra de alimentos orgánicos. Es decir, una persona preocupada por su salud, con altos niveles de estudio, con buena situación laboral e interesada en productos que informen sobre su composición, tiene mayor probabilidad de adquirir habitualmente alimentos ecológicos.

Los hallazgos de la investigación presentan diversas implicaciones para las empresas que producen y comercializan alimentos orgánicos. Por 
un lado, permiten revelar el perfil del consumidor habitual, las características socioeconómicas y motivaciones determinantes que explican su frecuencia de consumo. Y por otro, permiten comprender el valor que posee la certificación y la información nutricional que entregan del producto. Todo lo anterior supondría información clave para el diseño de estrategias comerciales efectivas que otorguen mayor competitividad a la oferta productiva empresarial actual.

Para futuras investigaciones se sugiere aplicar el estudio para diferentes tipos de alimentos orgánicos, a fin de verificar y contrastar la consistencia de los hallazgos de acuerdo con categorías específicas de productos. También sería recomendable replicar longitudinalmente la evolución de los factores que determinan la frecuencia de compra, de manera de identificar patrones de conducta incorporando nuevas dimensiones demográficas, culturales y de personalidad de los consumidores. Finalmente, se sugiere medir la importancia relativa de los atributos considerados relevantes para la decisión de compra de alimentos orgánicos, como la certificación y el precio. De este modo, se cuantificaría el peso relativo que tienen dichos atributos en las preferencias de los consumidores y la disposición a pagar por su inclusión.

\section{Literatura Citada}

Andrade, C.; Ayaviri, D.

2018. Demanda y consumo de productos orgánicos en el Cantón Riobamba, Ecuador. Información Tecnológica, 29(4): 217-226.

Aslihan Nasir, V.; Karakaya, F.

2014. Consumer segments in organic foods market. Journal of Consumer Marketing, 31(4): 263-277.

Auriol, E.; Schilizzi, S.

2015. Quality signaling through certification in developing countries. Journal of Development Economics, 116: 105-121.

Bryła, P.

2016. Organic food consumption in Poland: Motives and barriers. Appetite, 10: 737-746.

Cea-Valencia, J.; Fernández-Robin, C.; Santander-Astorga, P.;

Soto-Araya, D.; Yáñez-Martínez, D.

2016. Comportamiento del consumidor chileno frente a productos de empresas B: Análisis de percepción de precio e intención de compra. $M b r, 9(2)$ : 10-16.

Díaz, A.; Pérez, A.; Hernández, J.

2015. Caracterización del consumidor de productos orgánicos en la ciudad de Toluca, Méx. Revista Mexicana de Agronegocios, 36(1): 1178-1187.

Díaz, $\mathrm{P}$.

2017. Las tendencias que impulsan a los orgánicos en Chile. El Mercurio (Santiago, Chile). 2 de octubre 2017. Disponible: http://www.economiaynegocios.cl/noticias/ noticias.asp?id=402445. Consultado: 06/ene/2019.

Ditlevsen, K.; Sandøe, P.; Lassen, J.

2019. Healthy food is nutritious, but organic food is healthy because it is pure: The negotiation of healthy food choices by Danish consumers of organic food. Food Quality and Preference, 71: 46-53.

Higuchi, A.

2015. Características de los consumidores de productos orgánicos y expansión de su oferta en Lima. Apuntes, 42(77): 57-89.

Hwang, J.; Chung, J.

2019. What drives consumers to certain retailers for organic food purchase: The role of fit for consumers' retail store preference. Journal of Retailing and Consumer Services, 47: 293-306.
Ibáñez, F.

2019. El floreciente mercado de los alimentos orgánicos. Pauta.Cl (Santiago, Chile). 14 de marzo 2019. Disponible: https://www.pauta.cl/negocios/bloomberg/el-florecientemercado-de-los-alimentos-organicos. Consultado: 6/ $\mathrm{abr} / 2019$.

Janssen, M.

2018. Determinants of organic food purchases: Evidence from household panel data. Food Quality and Preference, 68: 19-28.

López-Cano, L.; Restrepo-Mesa, S.

2014. Etiquetado nutricional, una mirada desde los consumidores de alimentos. Perspectivas en nutrición humana, 16(2): 145-158.

Lutterbeck, B.

2001. "Más sano y con sabor más natural” ¿Quiénes compran alimentos orgánicos y por qué? Comuniica Magazine, 5(17): 23-25.

Massey, M.; O'Cass, A.; Otahal, P.

2018. A meta-analytic study of the factors driving the purchase of organic food. Appetite, 125: 418-427.

Nandonde, F.A.; Kuada, J.

2016. Modern food retailing buying behaviour in Africa: the case of Tanzania. British Food Journal, 118(5): 1163-1178.

Ozguven, $\mathrm{N}$.

2012. Organic Foods Motivations Factors for Consumers. Procedia - Social and Behavioral Sciences. 62: 661-665.

Parga-Dans, E.; Alonso, P.

2017. 'Marketing quality' in the food sector: Towards a critical engagement with the 'quality turn' in wine. Geoforum, 85: 5-8.

Rana, J.; Paul, J.

2017. Consumer behavior and purchase intention for organic food: A review and research agenda. Journal of Retailing and Consumer Services, 38: 157-165.

Singh, A.; Verma, P.

2018. Factors influencing Indian consumers' actual buying behaviour towards organic food products. Journal of Cleaner Production, 167: 473-483.

Stanton, J.; Cook, L.

2019. Product knowledge and information processing of organic foods. Journal of Consumer Marketing, 36(1): 240-252. 
Stoleru, V.; Munteanu, N.; Istrate, A.

2019. Perception Towards Organic vs. Conventional Products in Romania. Sustainability, 11(8): 2394.

Sweeney, F.; Vásquez, F.; Chavera, G.

2015. The concept organic in foods: a case study in the Azapa Valley, Chile. Idesia (Arica), 33(4): 121-123.
Ureña, F.; Bernabéu, R.; Olmeda, M.

2008. Women, men and organic food: Differences in their attitudes and willingness to pay. A Spanish case study. International Journal of Consumer Studies, 32(1): 18-26.

Vasco, C.; Palacios, G.; Paspuel, S.

2015. Determinantes socioeconómicos del consumo de productos ecológicos en Quito. Siembra, 2(2015): 23-28. 
